\title{
Editorial: Pharmacotherapy of Anxiety Disorders: Promises and Pitfalls
}

\author{
Amir Garakani ${ }^{1,2,3 *}$, Rafael C. Freire ${ }^{4}$ and James W. Murrough ${ }^{1,5}$ \\ ${ }^{1}$ Department of Psychiatry, Icahn School of Medicine at Mount Sinai, New York, NY, United States, ${ }^{2}$ Silver Hill Hospital, New \\ Canaan, CT, United States, ${ }^{3}$ Department of Psychiatry, Yale University School of Medicine, New Haven, CT, United States, \\ ${ }^{4}$ Department of Psychiatry and Centre for Neuroscience Studies, Queen's University, Kingston, ON, Canada, ${ }^{5}$ Department of \\ Neuroscience, Icahn School of Medicine at Mount Sinai, New York, NY, United States
}

Keywords: anxiolytic, novel medications, panic, social anxiety, specific phobia, generalized anxiety, PTSD, psychopharmacology

\section{Editorial on the Research Topic}

Pharmacotherapy of Anxiety Disorders: Promises and Pitfalls

Despite being among the most common psychiatric disorders worldwide, and a leading cause of disability including work and school absences, anxiety disorders have been relatively under-represented in recent research of novel pharmacologic agents, compared to major depressive disorder (MDD) and schizophrenia. Panic disorder (PD), generalized anxiety disorder (GAD), and social anxiety disorder (SAD) are commonly treated with either medications and/or psychotherapy, while specific phobias (SP) are usually treated with behavioral therapy alone. While there is support for certain forms of psychotherapy to treat anxiety disorders, there remains concern about lower efficacy of psychotherapies compared to medications (1), and incomplete treatment response, and evidence that patients with certain anxiety disorders, especially GAD and SAD, have high rates of recurrence $(2,3)$. Most research of medications of anxiety disorders have been focused on the gamma aminobutyric acid (GABA), serotonin and norepinephrine systems. The first-line medications approved by the United States Food and Drug Administration (FDA) for treatment of $\mathrm{PD}, \mathrm{GAD}$, and SAD are selective serotonin reuptake inhibitors (SSRIs) and serotonin norepinephrine reuptake inhibitors (SNRIs) while benzodiazepines, which are GABA-A receptor agonists, are also approved for treatment either as monotherapy or adjunctive treatment for anxiety (4-7). Despite the promise of newer serotonergic agents, or antipsychotics, or GABAergic drugs (like pregabalin and gabapentin), there has not been an FDA-approved medication for anxiety disorders since duloxetine, an SNRI, was approved for GAD in 2007 (8).

The purpose of this Research Topic was to collect original papers and review articles exploring promising novel medications on the pipeline for anxiety disorders, primarily GAD, PD, SAD and SP, after first reviewing the current state of psychopharmacological treatments available. The topic aimed to explore more unique pathways for targeting treatment response in anxiety disorders, including the glutamate system, neurosteroids, the hypothalamic-pituitary-adrenal (HPA axis), neuropeptides, cannabinoids, and phytochemicals.

Lijffijt et al. reported a protocol for a randomized, placebo-controlled proof-of-mechanism trial of a n-methyl-d-aspartate (NMDA) receptor antagonist lanicemine for 24 adults with symptoms of post-traumatic stress disorder (PTSD) (as measured by the Clinician Administered PTSD Scale (CAPS)]. The study was included in this Research Topic despite being a trial on participants with PTSD symptoms because of its potential application to anxiety disorders. In the protocol, 
participants are to receive 5 days of intravenous (IV) injections of lanicemine or placebo and be monitored with anxietypotentiated startle and CAPS scores. Lijffijt et al. are building upon previous research of another NMDA antagonist, ketamine, which has also been tested using IV infusions in patients with treatment-resistant depression (9-13), comorbid PTSD and MDD (14), and randomized controlled trials showing potential efficacy in PTSD as well $(15,16)$. There will be great interest in the results of this clinical study when it is concluded.

Given the heightened interest in NMDA receptor antagonists for depression and PTSD, Nasir et al. provided a review of glutamate, the principal excitatory neurotransmitter of the central nervous system (CNS), and its interactions with GABA, the primary CNS inhibitor, in anxiety disorders. Building from an overview of circuitry and receptor pathways, and a review of preclinical research describing the role glutamate plays in anxiety, Nasir et al. then describe the current state of glutamatergic and GABAergic drug research in anxiety disorders. The GABA modulators discussed in the paper, most of which are clinically used as anticonvulsants (levetiracetam, topiramate, tiagabine, and valproic acid), have limited support due the absence of larger, randomized, double-blind, placebo-controlled trials, with the exception of pregalabin, which was approved for GAD in Europe in 2006 (8). Glutamate modulators like ketamine, memantine, dcycloserine, n-acetylcysteine, and riluzole, have shown promise in open-label or small, controlled trials but there are few if any larger-scale studies.

Understanding stress response and hormone regulation may provide further clarity about newer pharmacological treatments, and Tafet and Nemeroff explored how the HPA axis plays a role in anxiety and stress. The review paper discusses the neurobiology of the HPA axis, serotonergic and norepinephrine systems and how traditional treatments for anxiety disorders, like tricyclic antidepressants (TCAs), SSRIs, and benzodiazepines may normalize hyperactivity of the HPA axis. Tafet and Nemeroff cite evidence that these antidepressants may modulate glucocorticoid receptors in the brain and benzodiazepines may have an inhibitory effect on corticotrophin releasing factor (CRF). Taken together, the findings support an expansion of research beyond serotonin and norepinephrine and may spur exploration of novel agents targeting the HPA axis directly.

\section{REFERENCES}

1. Chen T-R, Huang H-C, Hsu J-H, Ouyang W-C, Lin K-C. Pharmacological and psychological interventions for generalized anxiety disorder in adults: a network meta-analysis. J Psychiatr Res. (2019) 118:73-83. doi: 10.1016/j.jpsychires.2019.08.014

2. Bystritsky A. Treatment-resistant anxiety disorders. Mol Psychiatry. (2006) 11:805-14. doi: 10.1038/sj.mp.4001852

3. Roy-Byrne P. Treatment-refractory anxiety; definition, risk factors, and treatment challenges. Dialogues Clin Neurosci. (2015) 17:191-206. doi: 10.31887/DCNS.2015.17.2/proybyrne

4. Bandelow B, Reitt M, Röver C, Michaelis S, Görlich Y, Wedekind D. Efficacy of treatments for anxiety disorders: a meta-analysis. Int Clin Psychopharmacol. (2015) 30:183-92. doi: 10.1097/YIC.0000000000000078
Finally, the Topic Editors, Garakani et al., along with other authors, presented an overview of the current state of treatment of anxiety disorders, which is discussed above, followed by a review of novel pharmacologic treatments for PD, GAD, $\mathrm{SAD}$, and SP. Several serotonergic agents that were originally investigated for depression have been studied and showed potential, but these trials did not lead to FDA approval and there are limited ongoing investigations. There are also trials of psilocybin and lysergic acid diethylamide (LSD) for anxiety but primarily in those with life-threatening diseases (like cancer) $(17,18)$. Ketamine has been studied in a randomized controlled trial in SAD but there are no ongoing trials in anxiety disorders (19). Studies of d-cyloserine in anxiety have mostly been negative. There are few neuropeptides under study for anxiety as well and trials of CRF antagonists have not shown efficacy in anxiety disorders. Research on cannabinoids for anxiety have also been disappointing and shown possible worsening of symptoms, particularly with Delta-9-tetrahydrocannabinol (THC). The primary areas of promise are the neurosteroid PH94B, an intranasally administered aerosol, which underwent two randomized controlled trials for acute $\operatorname{SAD}(20,21)$, and the growing research on phytochemicals including kava.

Taken as a whole, the Research Topic did in fact show both promises and disappointments in the research of novel pharmacotherapeutics for anxiety disorders. As expected, the pathways and novel compounds hypothesized to treat anxiety were hampered by lack of efficacy (perhaps due to poor study design), small sample sizes or other mitigating factors. With any hope, this Topic should not discourage ongoing exploration beyond serotonin, norepinephrine and GABA, and instead bolster the efforts to expand on our understanding of the complex neurobiological pathways of anxiety and how to translate this work into novel agents that perhaps can complement the ongoing research into psychotherapeutic treatments of anxiety.

\section{AUTHOR CONTRIBUTIONS}

AG wrote the original draft of the manuscript. AG, RF, and JM contributed to critical manuscript revisions. All authors reviewed and approved the final draft of the manuscript and made substantial contributions to this study.
5. American Psychiatric Association. Practice Guideline for the Treatment of Patients With Panic Disorder. Washington, DC (2009).

6. National Institute for Health and Care Excellence (NICE). Generalised Anxiety Disorder and Panic Disorder in Adults: Management. Available online at: https://www.nice.org.uk/guidance/cg113 (accessed January 28, 2021).

7. National Institute for Health and Care Excellence (NICE). Social Anxiety Disorder: Recognition, Assessment and Treatment. Available online at: https:// www.nice.org.uk/guidance/cg159 (accessed January 28, 2021).

8. Sartori SB, Singewald N. Novel pharmacological targets in drug development for the treatment of anxiety and anxiety-related disorders. Pharmacol Ther. (2019) 204:107402. doi: 10.1016/j.pharmthera.2019.107402

9. Murrough JW, Iosifescu DV, Chang LC, Al Jurdi RK, Green CE, Perez AM, et al. Antidepressant efficacy of ketamine in treatment-resistant major depression: a two-site randomized controlled trial. Am J Psychiatry. (2013) 170:1134-42. doi: 10.1176/appi.ajp.2013.13030392 
10. Murrough JW, Perez AM, Pillemer S, Stern J, Parides MK, aan het Rot $\mathrm{M}$, et al. Rapid and longer-term antidepressant effects of repeated ketamine infusions in treatment-resistant major depression. Biol Psychiatry. (2013) 74:250-6. doi: 10.1016/j.biopsych.2012.06.022

11. Singh JB, Fedgchin M, Daly EJ, De Boer P, Cooper K, Lim P, et al. A doubleblind, randomized, placebo-controlled, dose-frequency study of intravenous ketamine in patients with treatment-resistant depression. Am J Psychiatry. (2016) 173:816-26. doi: 10.1176/appi.ajp.2016.16010037

12. Phillips JL, Norris S, Talbot J, Birmingham M, Hatchard T, Ortiz A, et al. Single, repeated, and maintenance ketamine infusions for treatmentresistant depression: a randomized controlled trial. Am J Psychiatry. (2019) 176:401-9. doi: 10.1176/appi.ajp.2018.18070834

13. Fava M, Freeman MP, Flynn M, Judge H, Hoeppner BB, Cusin C, et al. Doubleblind, placebo-controlled, dose-ranging trial of intravenous ketamine as adjunctive therapy in treatment-resistant depression (TRD). Mol Psychiatry. (2020) 25:1592-603. doi: 10.1038/s41380-018-0256-5

14. Albott CS, Lim KO, Forbes MK, Erbes C, Tye SJ, Grabowski JG, et al. Efficacy, safety, and durability of repeated ketamine infusions for comorbid posttraumatic stress disorder and treatment-resistant depression. J Clin Psychiatry. (2018) 79:17m11634. doi: 10.4088/JCP.17m11634

15. Feder A, Parides MK, Murrough JW, Perez AM, Morgan JE, Saxena S, et al. Efficacy of intravenous ketamine for treatment of chronic posttraumatic stress disorder: a randomized clinical trial. JAMA Psychiatry. (2014) 71:681-8. doi: 10.1001/jamapsychiatry.2014.62

16. Feder A, Costi S, Rutter SB, Collins AB, Govindarajulu U, Jha MK, et al. A randomized controlled trial of repeated ketamine administration for chronic posttraumatic stress Disorder. Am J Psychiatry. (2021) 178:193202. doi: 10.1176/appi.ajp.2020.20050596

17. Fuentes JJ, Fonseca F, Elices M, Farré M, Torrens M. Therapeutic use of LSD in psychiatry: a systematic review of randomized-controlled clinical trials. Front Psychiatry. (2019) 10:943. doi: 10.3389/fpsyt.2019.00943

18. Weston NM, Gibbs D, Bird CIV, Daniel A, Jelen LA, Knight G, et al. Historic psychedelic drug trials and the treatment of anxiety disorders. Depress Anxiety. (2020) 37:1261-79. doi: 10.1002/da.23065

19. Taylor JH, Landeros-Weisenberger A, Coughlin C, Mulqueen J, Johnson JA, Gabriel D, et al. Ketamine for social anxiety disorder: a randomized, placebo-controlled crossover trial. Neuropsychopharmacology.

(2018) 43:325-33. doi: 10.1038/npp.2017.194

20. Liebowitz MR, Salman E, Nicolini H, Rosenthal N, Hanover R, Monti L. Effect of an acute intranasal aerosol dose of $\mathrm{PH} 94 \mathrm{~B}$ on social and performance anxiety in women with social anxiety disorder. Am J Psychiatry. (2014) 171:675-82. doi: 10.1176/appi.ajp.2014.12101342

21. Liebowitz MR, Hanover R, Draine A, Lemming R, Careri J, Monti L. Effect of as-needed use of intranasal PH94B on social and performance anxiety in individuals with social anxiety disorder. Depression Anxiety. (2016) 33:1081-9. doi: 10.1002/da.22546

Conflict of Interest: JM disclosures: In the past 5 years, JM has provided consultation services and/or served on advisory boards for Allergan, Boehreinger Ingelheim, Clexio Biosciences, Fortress Biotech, FSV7, Global Medical Education (GME), Impel Neuropharma, Janssen Research and Development, MedavanteProphase, Novartis, Otsuka, and Sage Therapeutics. JM was named on a patent pending for neuropeptide $\mathrm{Y}$ as a treatment for mood and anxiety disorders and on a patent pending for the use of ezogabine and other KCNQ channel openers to treat depression and related conditions. The Icahn School of Medicine (employer of JM) was named on a patent and has entered into a licensing agreement and will receive payments related to the use of ketamine or esketamine for the treatment of depression. The Icahn School of Medicine is also named on a patent related to the use of ketamine for the treatment of PTSD. JM was not named on these patents and will not receive any payments.

The remaining authors declare that the research was conducted in the absence of any commercial or financial relationships that could be construed as a potential conflict of interest.

Copyright (C) 2021 Garakani, Freire and Murrough. This is an open-access article distributed under the terms of the Creative Commons Attribution License (CC BY). The use, distribution or reproduction in other forums is permitted, provided the original author(s) and the copyright owner(s) are credited and that the original publication in this journal is cited, in accordance with accepted academic practice. No use, distribution or reproduction is permitted which does not comply with these terms. 